\title{
CROSSING OVER OHNE CHIASMATYPIE?
}

\author{
RICHARD GOLDSCHMIDT \\ Kaiser Wilhelm-Institut fïr Biologie, Berlin \\ [Eingegangen am 28. November 1916]
}

1

Es ist wohl nicht zu viel gesagt, wenn man behauptet, dass Morgans und seiner Mitarbeiter Untersuchungen an Drosophila die wichtigste Bereicherung der Vererbungslehre in den letzten Jahren darstellt. Sie sind ein Musterbeispiel für ein zielbewusstes Handinhandarbeiten von Vererbungsexperiment und cytologischer Forschung und ihre Hauptergebnisse gehören jetzt schon zum wichtigsten Schatz unserer Wissenschaft. Wenn irgendwelche Arbeiten im Stande waren, auch die letzten Zweifler von der Richtigkeit der Chromosomenlehre der Mendelschen Vererbung zu überzeugen, so sind es diese. Diese Ueberzeugung soll uns aber nicht blind machen und verhindern, da Kritik anzulegen, wo es notwendig erscheint. Wenn dies im Folgenden geschehen soll, so tue ich es in der Ueberzeugung, dass die Tragweite der Morganschen Resultate nur erhöht werden kann, wenn sie von Folgeschlüssen befreit sind, die nicht notwendig sind, und so bestechend und geistreich sie auch sein mögen, einer weiteren Vertiefung der Erkenntnis dadurch schaden, dass sie sie in einer bestimmten, aber nicht notwendigen Richtung festlegen.

Der auffallendste und imposanteste Schluss aus Morgans Arbeiten ist natürlich der, dass er aus den Zahlenverhältnissen der "crossover" Klassen in den Zuchten auf die relative Lage der körperlichen Aequivalente der Erbfaktoren im Chromosom schliessen kann. Und dieser Schluss wieder basiert auf der Annahme, dass der in seinen Zahlenverhältnissen typische Austausch zwischen den homologen Chromosomen durch den Austausch von Chromosomensegmenten im Gefolge einer Chiasmatypie gegeben wird. MoRGan selbst ist sich natürlich der Tatsache bewusst, dass die Annahme von Janssens Theorie der Chiasmatypie, so weit die cytologischen Tatsachen in Betracht kommen, ziemlich in der Luft schwebt. Man könnte ihm trotzdem in der Verwendung dieser Hypothese, angesichts ihres Erklärungswerts, zustimmen, wenn sie nötig wäre. Aber das ist nicht der Fall. Und nicht nur dies, es erscheint uns sogar, dass die Chiasmatypiehypothese und die sich daraus ergeben- 
den Konsequenzen in bezug auf die Lagerung der Faktoren im Chromosom ein Nachteil ist. Sie legt unsere Vorstellungen auf einen relativ einfach erscheinenden, grobsinnlichen Vorgang fest und belastet uns damit mit einer Kette, die unter Umständen verhindert, dass aus den gleichen Tatsachen noch weiterreichende Schlüsse gezogen werden können. Sie täuscht uns die Erreichung einer zweiten Stufe der Erkenntnis vor, während in Wirklichkeit nur die erste erreicht ist, und aus den zahllosen gleichberechtigten Möglichkeiten für die zweite, nur eine einzige, leicht sinnlich vorstellbare, heraus gegriffen ist. Es wird sogleich klar werden, was wir damit meinen.

Die erste Stufe, die unseres Erachtens durch Morgans Experimente mit Sicherheit erreicht ist, ist der Nachweis, dass Faktoren zwischen den Chromosomen eines Paares ausgetauscht werden können und dass das Mass, in dem sie ausgetauscht werden, für je zwei oder mehr gegebene Faktoren unter gleichen äusseren Bedingungen ein typisches ist, variierend zwischen vollständiger "Linkage" oder Nichtaustausch und freier Spaltung oder beliebigem Austausch. Die zweite Stufe der Erkenntnis wäre dann der Nachweis der Kräfte, die diès quantitativ verschiedenartige Verhältnis des Austausches bedingen. Für Morgan ist es die Chiasmatypie und die Entfernung der Faktoren im Chromosom, also morphologische Lagebeziehungen, die dafür verantwortlich sind. Dies ist der Schluss, den wir als unnötig nachweisen wollen, indem wir zeigen, dass für die Erklärung jener quantitativen Verhältnisse die gleichen Kräfte ausreichen, die überhaupt beim Aufbau eines Chromosoms aus Faktoren am Werk sein müssen, welcher Art sie auch seien. An die Stelle jener Schlussfolgerung wollen wir eine viel allgemeinere setzen, von der Morgans Hypothese nur ein grobsinnlich gefasster Spezialfall ist. Er sieht in den unbekannten, quantitativ bestimmten Kräften des Austauschs eine entsprechende Entfernung der. Faktoren im Chromosom. Es ist aber doch klar, dass man jede Proportion geometrisch als Entfernungen auf einer Geraden darstellen kann. Wenn diese Darstellung also im gegebenen Fall stets mit den Tatsachen übereinstimmt, so beweist das nicht etwa, dass nun wirklich Entfernungen auf einer Geraden hinter der Erscheinung als Ursache stehen, so nde rn es beweist nur, dass irgendwelche Kräfte im Spiel sind, deren relativer Effekt als Entfernungen a uf e iner Geraden dargestellt werden können. Wir glauben, dass es sehr wichtig ist, dies uns klar zı machen; denn während Morgans Hypothese nur den allereinfachsten denkbaren Spezialfall in 
Betracht zieht, erlaubt jene allgemeine Fassung nicht nur alle möglichen anderen Vorstellungen, sondern lässt auch die wichtige Möglichkeit zu, später einmal aus den beobachteten Zahlenverhältnissen Schlüsse auf die wirkliche Art der wirkenden Kräfte ziehen zu können, ähnlich wie man aus den bei Temperatur- und Variationsversuchen an Embryonen gewonnenen Zahlen auf den chemischen Charakter der zu Grunde liegenden Reaktionen schliessen konnte. Wir wollen somit beweisen-was eigentlich auch ohne besonderen Beweis klar sein sollte-dass a us den Crossover-Experimenten nurdies folgt, dass das Mass des Crossing overder Ausdruckirgendeiner quantitativ variabeln Kräfte wirkung ist, dief die Zugehörigkeit eines Determinanten zu einem der Chromosomenpartnerverantwortlich ist, eine Kraft deren relative zahlenmässige Wirkungen nat ürlich a uch geometrisch als Abschnitte einer Geraden dargestellt werden können.

\section{II}

Morgans Schlussfolgerungen aus seinen Versuchen, und zwar sowohl die, die uns hier beschäftigen, wie alle anderen cytologischer Natur, basieren auf einer Reihe von Annahmen, die ein jeder Anhänger der Chromosomenlehre der Vererbung machen muss. Ueber sie wollen wir uns zuerst einmal klar werden. Da ist zunächst die Annahme der Individualität der Chromosomen, ohne 'die ja überhaupt eine Parallele zwischen Chromosomenverteilung und Faktorenspaltung nicht durchführbar ist. Sodann haben wir die Annahme, dass in jedem Chromosom mehrere und verschiedenartige Erbfaktoren enthalten sind. Für ihre Richtigkeit bringen ja gerade Morgans Untersuchungen so überzeugendes Beweismaterial bei. Sodann muss angenommen werden, dass die Lagerung der Teilchen, die den Erbfaktoren entsprechen, innerhalb des Chromosoms eine typische ist, ob wir sie uns nun linear angeordnet vorstellen oder nicht. Denn sonst wäre ein geordnetes "Crossing over" überhaupt nicht denkbar. Daraus folgt aber noch eine andere wichtige Annahme. Während der Kernruhe werden die Chromosomen körperlich desintegriert. Bei der nächsten Teilung finden sie sich aber wieder unter Wahrung der Individualität vor. Es müssen also irgend welche Kräfte im Spiel sein, die bei der Bildung der Chromosomen immer wieder jedem PartikelchenErbfaktor seinen Platz im richtigen Chromosom und am richtigen Platz 
anweisen. Ob dieś nun für gewöhnlich ausgesprochen wird oder nicht, sicher ist, dass jede Chromosomenhypothese auf dieser Vorstellung basiert.

Und nun bleiben wir einmal bei diesem letzteren Punkt. Da ist es zunächst klar, dass wir über die Art der Kräfte, die die richtige Sammlung der Partikelchen zum Chromosom bedingen, nichts wissen. Es mögen chemische Affinitäten sein, es mögen Wirkungen der Massenkraft sein, es mögen grobmechanische Dinge sein. Aber unter allen Umständen müssen sie spezifisch und typisch für jedes gegebene PartikelchenErbfaktor sein. Ist dies schon für irgend eine geordnete Lagerung der Teilchen im Chromosom nötig, so ist es gewiss so für eine geordnete lineare Lagerung, mit der auch Morgan arbeitet. Für unsre weiteren Auseinandersetzungen ist es gänzlich gleichgiltig, wie wir uns die typische, geordnete Lagerung vorstellen. Und so ist es das Einfachste, dass wir uns auch die Dinge als lineare Anordnung der Partikelchen versinnlichen, da es die einfachste graphische Darstellung erlaubt; auch der Chemiker benutzt ja flächenhaft angeordnete Symbole anstatt der verwickelteren stereometrischen Vorstellung, ohne sie deshalb als eine Realität zu nehmen. Wenn nun also die Partikelchen sich zu einer typischen Reihe spezifischer Anordnung immer wieder zusammenfinden sollen, so müssen, wie gesagt, die wirkenden Kräfte für jedes einzelne in Bezug auf seine Nachbarschaft typisch sein. Wenn wir uns das versinnbildlichen wollen, so können wir die Kräfte, die einem Partikel die Lage neben einem andern und nur neben diesem anweist, als eine Wirkung bestimmter Quantität darstellen und graphisch könnten wir uns dann die Festlegung eines bestimmten Platzes im Chromosom für ein Partikelchen so veranschaulichen wie es Fig. I wiedergiebt. Die Kraft, die ein Partikelchen-Erbfaktor an sein Chromosom kettet ist durch einen rechteckigen Anker wiedergegeben, dessen Grösse der Quantität dieser Kraft entspricht. (Wir "verankern" den Faktor an seinem Chromosom im Interesse der graphischen Darstellung; sachlich kann das aber eben so gut die Verankerung an seinem Nachbar oder sonst etwas bedeuten). In der Figur I sind somit die Erbfaktoren $A B C D$ enthalten, die ihre richtige Lagerung in dem betreffenden Chromosom-die hier linear dargestellt ist aber ebenso gut irgend eine stereometrische Form haben könnteden spezifischen Kräften von der spezifischen Quantität und Qualität $w x y z$ verdanken. Wenn wir nun das entsprechende Chromosom einer Form darzustellen hätten, die an Stelle von $B$ nur $b$ enthält, so könnten wir es entweder unter wörtlicher Auslegung der presence- 

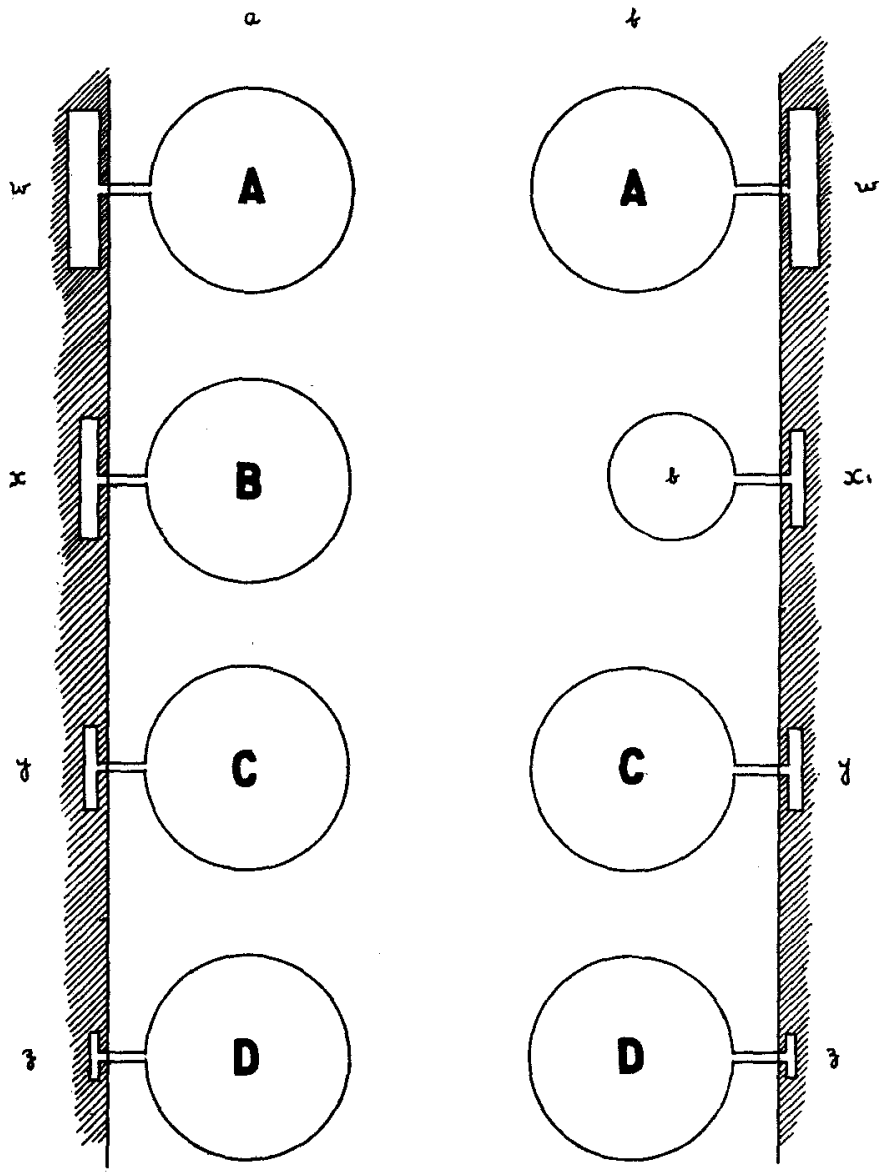

FiG. I

absence Theorie so machen, dass hier $B$ fehit, oder atch einen andersartigen Faktor $b$ einfügen, dessen Andersartigkeit sich auch in seiner andersartigen und spezifischen Verankerung zeigt also $x_{1}$ statt $x$. Sachlich ist es gleichgiltig, welche Darstellung wir wählen, denn schliesslich sind ja beide nur differente Anschauungsformen von presence-absence. Die letztere ist bequemer zu handhaben und daher in Fig. I $b$ angewandt. Wenn wir nun den Bastard $B b$ vor uns haben, so werden also auch bei ihm bei der Chromosomenbildung die Faktoren $B$ und $b$ an ihren richtigen Platz in dem richtigen väterlichen oder mütterlichen Chromosom sich einfinden und Fig. I wäre dann eine Darstellung eines Chromosomenpaares dieses Bastards. Wie schon gesagt, hier haben wir 
nicht das geringste $\mathrm{Neue}$ a usgesagt, sonderneinfach die selbstverständlichen Voraussetzungen der Chromosomenlehre uns graphisch klar $\mathrm{gem}$ a cht.

Daraus folgt nun ohne Weiteres das Folgende: Sind die Kräfte $x$ und $x_{1}$ quantitativ so verschieden, dass sie nicht vertauscht werden können, so können die Faktoren $B$ und $b$ stets nur wieder sich in ihrem ursprünglichen Chromosom einfinden; im Vererbungsversuch erschienen sie also vollständig "linked" mit dem Rest der Faktoren. Wären die Kräfte $x, x_{1}$ aber so ähnlich, dass sie vollständig freien Austausch der Faktoren gestatteten, die sich somit beliebig in jedem der beiden elterlichen Chromosomen an dem betreffenden Platz einfinden könnten, so müssten $B b$ eine reine Mendelspaltung zeigen, die nicht von einer Spaltung von in differenten Chromosomen gelagerten Faktoren zu unterscheiden wäre. Wären endlich aber die Kräfte $x, x_{1}$ variabel und ein Überschneiden der Curven fände statt, so hätten nur die in dem gemeinsamen Kurvenbezirke gelegenen Fälle die Möglichkeit ihren Platz in dem einen oder anderen Chromosom zu finden, d.h., zu cross over. Diese drei Möglichkeiten können wir uns an den nebenstehenden Variations kurven für die Kräfte $x, x_{1}$ ohne Weiteres klar machen (Fig. 2 ),
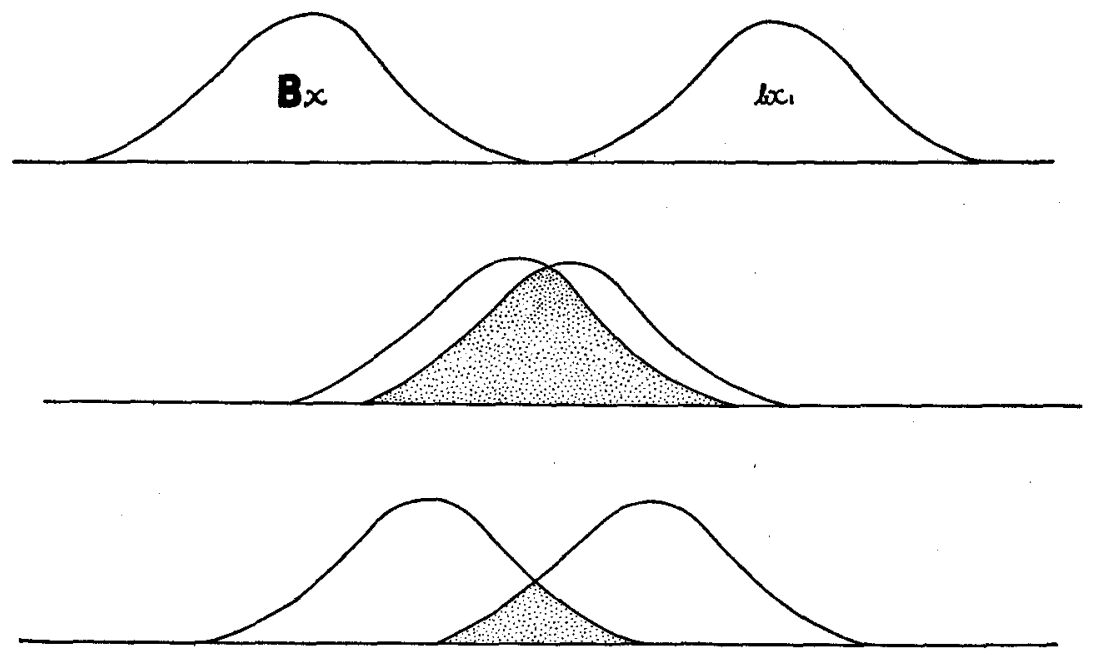

FIG. 2

wobei natürlich die einfachste Annahme über die Art der Variation gemacht ist. Die Zahl der Fälle des Hinüberkreuzens eines Faktors wäre aber proportional der Grösse des gemeinsamen Kurvenbezirks, und bei 
zwei Faktoren, die allein ja das Resultat erkennen liessen, proportional der Combination beider Kurvenbezirke. Und wenn, wie selbstverständlich, die Werte $x, x_{1}$, etc., zu den typischen Qualitäten der Faktoren $B$, etc., gehören, so muss auch in diesem Fall für jede Faktorenkombination der Prozentsatz des Hinüberkreuzens unter gleichen Bedingungen konstant sein. So zeigt es sich, dass die ganze Erscheinung des Crossing over eine logische Konsequenz aus der Individualität der Chromosomen ist, somit keine weitere Hilfshypothese zu ihrer Erklärung nötig ist. Es zeigt ferner, dass der typische Prozentsatz der crossover Klassen ganz allgemein der Ausdruck einerentsprechend typischen Verschiedenheit der den Chromosomena $\mathrm{f}$ bau a us den Einzelfaktoren bedingenden Kräfte ist; und somit gar kein Bedürfnisvorliegt, an Stelle der Kräfterelationen, deren Wirkung natürlich auch geometrisch als Abschnitte einer Geraden dargestellt werden können, die ganz bestimmte Vorstellung der Chiasmatypie und räumlichen Entfernung zu setzen.

Hier könnte nun noch die Frage aufgeworfen werden, ob ein derartiger Faktorenaustausch nicht eben so gut in den diploiden Keimbahnzellen stattfinden müsse, wie in den Geschlechtszellen vor den Reifeteilungen. A priori lässt sich dies in der Tat nicht leugnen. Sachlich würde das aber nichts ändern, sondern nur bedeuten, dass das Mass des Crossover eine Funktion von zwei Variabeln, nämlich sowohl der betreffenden Verankerungskräfte als auch der Zahl der somatischen Keimbahnteilungen ist. In Fällen, in denen die Crossoverzahlen in verschiedenen Versuchen verschieden ausfallen (etwa bei Antirrhinum) mag tatsächlich diese Annahme die Erklärung liefern. Sonst ist sie unnötig und daher empfehlenswert nur mit einem einmaligen Austausch in der Synapsis und Wachstumsperiode zu rechnen.

Als wichtig erscheint in diesem Zusammenhang auch das folgende. Es ist bemerkenswert, dass bei Drosophila der Faktorenaustausch nur im weiblichen Geschlecht stattfindet, in anderen Beispielen nur im männlichen oder in beiden. Wenn der Austausch bedingt wird durch die transgredierende Variation zweier Kräfte, so sollte diese abhängig sein von Aussenfaktoren. Die Aussenwelt für die Chromosomen is zunächst der Kern. Tatsächlich gehen während der den Reifeteilungen vorausgehenden Wachstumsperiode im Keimblässchen Umwälzungen vor sich, 
die die Variationsursachen abgeben könnten. Das Crossing over fände dann bei dem Neuaufbau der Chromosomen zu den Reifeteilungen statt, und eventuelle Verschiedenheiten darin zwischen den Geschlechtern wären zu erklären aus den Bedingungen dieser Periode, die Variation begünstigen oder nicht. Damit stimmt auch die Tatsache überein, dass die Crossoverzahlen durch äussere und innere Einflüsse (Altern bei Drosophila) verändert werden können. Dies ist natürlich auch nur eine Hypothese, die für die Gesamtbetrachtung nicht wesentlich ist.

Um Missverständnisse zu vermeiden sei an dieser Stelle nochmals folgendes hervorgehoben. Es ist nicht meine Absicht, an Stelle von MorGANS Hypothese eine andere zu setzen, oder gar seine Darstellung als grobsinnliches Schema zu verwerfen, und dann durch Aufstellung eines anderen Schemas denselben Fehler $z \mathfrak{u}$ begehen. Das, was ich beweisen will, ist vielmehr, dass die Tatsachen auf Grund der von jedermann anerkannten Voraussetzungen der Chromosomenlehre verstanden werden können; dass dieselben Kräfte, die die Chromosomenindividualität bedingen, auch für die Erklärung des Crossing over ausreichen; ferner, dass wir für die Wirkung dieser unbekannten Kräfte verschiedene diagrammatische Darstellungen wählen können, die der graphische Ausdruck von Zahlenrelationen sind; und sodann, dass wir eine passende graphische Darstellung nicht mit der Wirklichkeit verwechseln sollen, da wir uns dadurch den Weg für weitere Erkenntnis versperren, besonders für eine solche physikalisch-chemischer oder dynamischer Natur.

\section{III}

Die hier entwickelten Anschauungen sind von mir schon mehrfach, für den Fachmann verständlich, angedeutet worden. JANssens Chiasmatypie-Theorie sollte ja eine Erklärung für die Möglichkeit des Vorhandenseins mehrerer spaltender Faktoren in einem Chromosom geben. Die crossover Theorie steht aber auf der gleichen Basis. Schon IgI I habe ich eine Idee entwickelt, wie das Vorhandensein mehrerer selbstständig spaltender Faktoren in einem Chromosom erklärt werden kann, der prinzipiell derselbe Gedankengang, wie der hier durchgeführte, zu Grunde liegt. Die damaligen Ausführungen hatten aber den Nachteil, dass sie mit der Annahme der end-to-end Conjugation verquickt waren und auch mit einer zu wörtlichen Auslegung der presence-absence Theorie. Sie haben daher auch keine weitere Beachtung gefunden.

Diese früheren Äusserungen haben wohl Morgan, Mulder, Sturtevant u. Bridges (I9I5) im Sinn, wenn sie von der Anschauung "einer 
Anzahl Genetiker" sprechen, ${ }^{1}$ die das Crossover mit dem Chromosomenaufbau aus Partikelchen in Zusammenhang bringen. Muller (1916) geht in seiner neuesten Arbeit etwas näher auf diesen Punkt ein. Da im Vorhergehenden und Folgenden alle von ihm angeführten Punkte ohnehin besprochen werden, brauchen wir hier nicht noch einmal darauf zurück zu kommen. Aber es erscheint doch wünschenswert, noch im Speziellen zu zeigen, dass tatsächlich die Versuchsergebnisse in der hier durchgeführten Weise erklärt werden können.

Nehmen wir an, wir hätten in einem Versuch zu tun mit den elterlichen Faktoren $A B$ und $a b$. Bei dem Aufbau der Chromosomen zur Reifeteilung gelangen also normaler Weise $A B$ und $a b$ in die homologen Chromosomen. Wenn die Kraft, die ihnen ihren Platz anweist bei $A$ und $a$ oder $B$ und $b$ sehr verschieden ist, tritt völlige "Linkage" ein. Ist. sie aber nur soweit verschieden, dass die Grenzfälle transgredieren, dann tritt Crossing over ein. Nehmen wir nun an, dass für die Faktoren $A$ a die elterliche Lage im Chromosom 3 mal so oft eintritt als das Ueberkreuzen, also unter 4 Fällen 3 normal sind; ferner, dass für die Faktoren $B b$ das gleiche Verhältnis 5: I sei. Dann wird, falls dies nur in einem Geschlecht stattfindet, das Verhältnis der elterlichen Kombinationen zu den crossover Klassen $A b$ und $a B$ gleich $16: 8$ sein oder 50 prozent. Wenn wir anstatt $3:$ I und $5:$ I allgemein setzen $p_{a}:$ I und $p_{b}:$, , so giebt uns die Formel $\frac{100\left(\mathrm{p}_{\mathrm{a}}+\mathrm{p}_{\mathrm{b}}\right)}{\left(\mathrm{p}_{\mathrm{a}}+\mathrm{I}\right)\left(\mathrm{p}_{\mathrm{b}}+\mathrm{I}\right)}=\mathrm{cr}$ die crossover Klassen für $A a B b$ in Prozent wieder. Empirisch gefundene Werte für cr könnten natürlich auf sehr verschiedenen Werten von $p_{a}$ und $p_{b}$ berthen. Wenn aber noch ein drittes Faktorenpaar $C c$ hinzu kommt, so legen die crossover Prozente für $A B, A C$ und $B C$ die Werte $\mathrm{p}_{\mathrm{a}} \mathrm{p}_{\mathrm{b}} \mathrm{p}_{\mathrm{c}}$ fest. Nehmen wir nun einmal eine Serie von Faktorenpaaren $A a B b C c D d$, etc., und setzen für sie angenommene Werte für $\mathrm{p}_{\mathrm{a}}, \mathrm{p}_{\mathrm{b}} \ldots$. , nämlich:

$$
\begin{aligned}
& \mathrm{p}_{\mathrm{a}}=3 \\
& \mathrm{p}_{\mathrm{b}}=4 \\
& \mathrm{p}_{\mathrm{c}}=\mathrm{I0} \\
& \mathrm{p}_{\mathrm{d}}=20 \\
& \mathrm{p}_{\mathrm{f}}=50 \\
& \mathrm{p}_{\mathrm{i}}=\mathrm{I000}
\end{aligned}
$$

und rechnen die danach zu erwartenden crossover Prozente für die verschiedenen Faktorenkombinationen aus, dann sind

1 Auch Stomps (I912) hat einmal ähnliche Äusserungen veröffentlicht. 


$$
\begin{array}{llll}
\operatorname{cr} A B=35.0 \% & \text { cr } B C=25.5 \% & \text { cr } C D=13.0 \% & \operatorname{cr} D F=6.5 \% \\
\operatorname{cr} A C=29.5 \% & \text { cr } B D=22.9 \% & \text { cr } C F=10.7 \% & \text { cr } D I=4.9 \% \\
\operatorname{cr} A D=27.4 \% & \text { cr } B F=21.2 \% & \text { cr } C I=9.9 \% & \text { cr } F I=2.1 \% \\
\operatorname{cr} A F=26.0 \% & \text { cr } B I=20.1 \% & & \\
\operatorname{cr} A I=25.0 \% & & &
\end{array}
$$

Wenn wir dann diese Werte graphisch nach Morgans Vorgang als Distanzen auf einer Geraden darstellen und dabei, wie MorgaN es tut, die kürzesten Strecken als massgebend nehmen, erhalten wir:

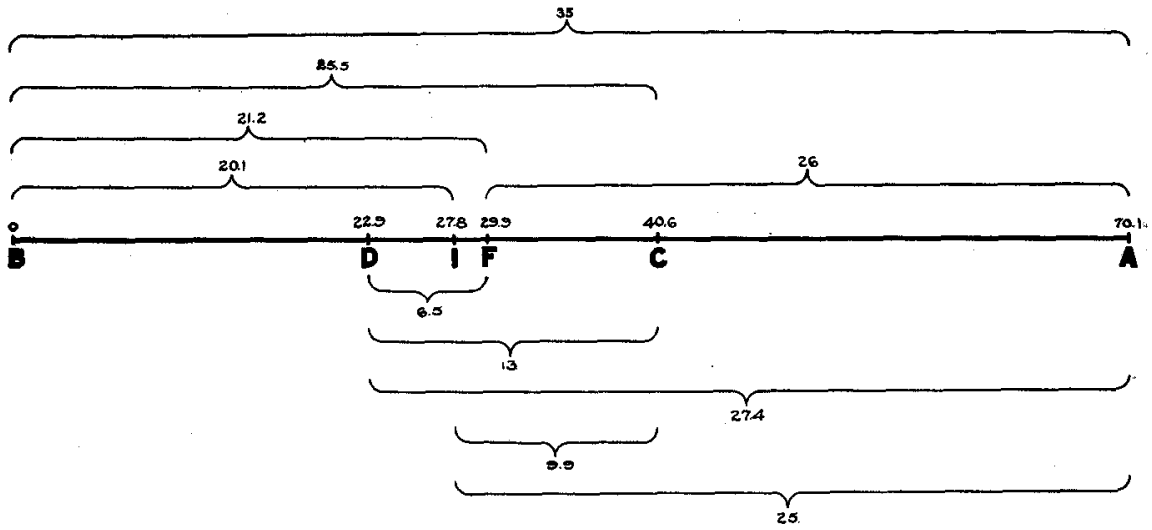

FIG. 3

Stellen wir nun daneben ein analoges Schema nach Sturtevants. (I9I3) Untersuchungen über die Drosophila-Faktoren $B, C O, P, R, M$ :

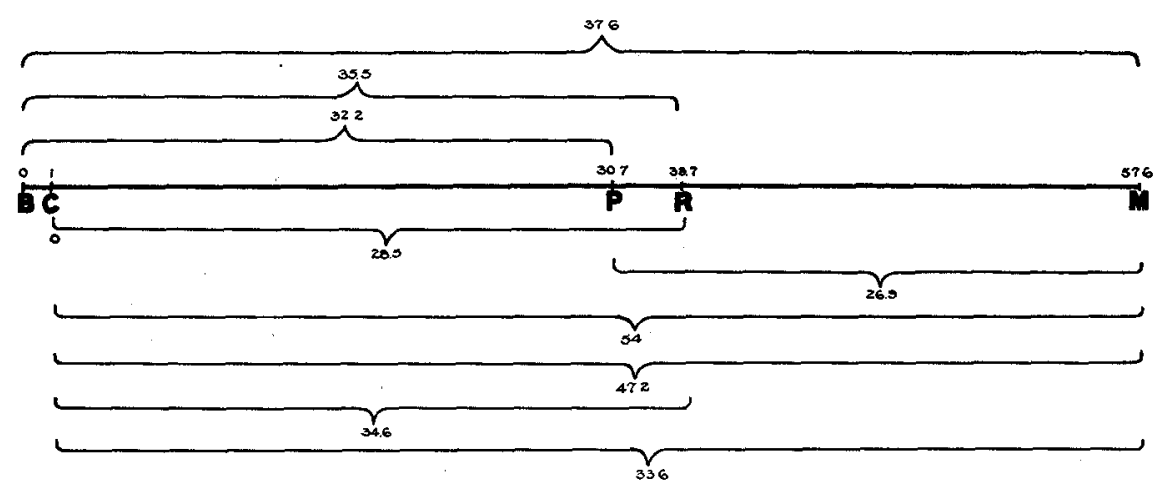

FIG. 4

Dies letztere soll die folgenden Gesetzmässigkeiten zeigen: Wenn es. sich um kleine Distanzen handelt, giebt die Addition zweier Teilstrecken ziemlich genau den für die ganze Strecke berechneten Wert, z.B., $B P=32.2, P R=3$ und $B R=35.5$. Analog wäre in unserem Schema. 
$B I=20 . \mathrm{I}, I F=2 . \mathrm{I}, B F=2 \mathrm{I} .2 ; A I=25.0, I D=4.9, A D=27.4$. Wenn es sich um grössere Distanzen handelt, giebt die Addition der Teilstrecken eine gröszere Summe als die Berechnung des Gesammtdistanz, z.B., $B, C O, P, R, M=57.6, B M=37.6$, oder $C O, P, M=56.6, C O M=54$ resp. 33.6 resp. 47.2. Dem steht gegenüber im ersten Schema $B D I F$ $C A=7$ o. I, $B A=35.0$; oder $B D I F C=40.6, B C=25.5$. Daneben finden sich aber noch allerlei nicht besonders hervorgehobene Inkongruenzen, z.B., bei Sturtevant $C R=28.5, C P=30$, also eine Summe kleiner als eines der Teilstücke. Ebenso $C M=33.6$ aber $C R=34.6$. Bei uns entspricht $\operatorname{dem} B D=22.9, B I=20$. I. $D a D I=4.9$ so würden wir, wenn nur diese 3 Werte bekannt wären, $I$ zwischen $B$ und $D$ setzen und es stimmte dann. Das Gleiche wäre aber der Fall, wenn Sturtevant nur die drei obigen Zahlen hätte. Ferner zeigt es sich, dass man verschiedene Werte für die gleiche Strecke bekommt bei verschiedener Art der Berechnung. So bei Sturtevant für $B M$ aus $B C+C P+P R+R M$ $=57.6$; aus $B R+R M=59.4 ;$ aus $B O+O M=48.4$; aus $B C+C M=$ 34.8, etc. Dem entspricht im ersten Schema für $B A$ ans $B D+D I+$ $I F+F C+C A=70 . \mathrm{I}$; aus $B I+I A=45 . \mathrm{I}$, aus $B C+C A=55.0$, etc.

In gleicher Weise könnte man natürlich auch ein double crossover Experiment berechnen und erhielte z. B. für den Versuch $B D C$ die Zahlen

$$
\begin{aligned}
& 67 \% \text { non-crossover } \\
& 10 \% \text { crossover } D C \\
& 20 \% \text { crossover } B D \\
& 3 \% \text { double crossover } B D C
\end{aligned}
$$

Es sei dies aber nicht weiter ausgeführt, einmal, weil wir glauben, dass die Sturtevant'schen Vergleichszahlen auf Grund einer falschen Formel berechnet sind, und sodann weil es, wie so oft schon hervorgehoben, gar nicht unsere Absicht ist, das hier benutzte Schema an Stelle des Morganschen setzen zu wollen.

MORGAN weist nun noch als besonders beweiskräftig auf die Tatsache hin--inzwischen von Muller im einzelnen ausgearbeitet,---dass bei der Spaltung von Bastarden von der Zusammensetzung $A B C D E F G a b c d e f g$ immer ganze Faktorengruppen beisammen bleiben, also meist single Crossing over, nur selten double und triple Crossing over eintritt. Eine Berechnung nach unserer Formel zeigt, dass auch dies als eine mathematische Konsequenz daraus abgeleitet werden kann, wie ja schon die Berechnung für das double Crossover zeigt. In Mullers-zweifellos höchst schwierigen-Experimenten findet sich bereits für triple Crossing 
over nur I Individuum. Nur bei viel grösseren Zahlen könnte man also die höheren crossover Klassen erwarten. Es braucht schliesslich wohl kaum hervorgehoben $z \mathfrak{u}$ werden, dass der Erklärungswert von MoRGANs Hypothese in Bezug auf multiplen Allelomorphismus und ähnliche Erscheinungen nicht dadurch berührt wird, wenn statt "Locus" Kräfterelation steht.

Zum Schluss dieses Abschnitts noch eine Bemerkung. Es könnte jemand auf den Gedanken kommen, nach meinen Auseinandersetzungen die Auflösungsformel zu berechnen und danach die Werte p der obigen Formel für die von MoRGAN gelieferten Zahlen zu berechnen, und dann vielleicht Inkongruenzen im Resultat als Widerlegung der hier vorgebrachten Anschauungen aufzuzeigen. Das wäre aber ein grobes Missverständnis. Ich habe, um meine These durchzuführen, möglichst einfache mathematische Voraussetzungen angenommen. Es liegt mir aber durchaus fern $z \mathfrak{l}$ behaupten, dass gerade diese der Wirklichkeit entsprechen. Im Gegenteil ist es ja meine Hoffnung, dass die Betrachtung der empirischen Zahlen umgekehrt einmal die richtige Formel für die zu Grunde liegenden Kräfte und damit ihre physikalisch-chemische Zuweisung ermöglichen werde. Das, um was es sich mir handelt, ist vielmehr, wie schon oben gesagt, $z \mathfrak{u}$ zeigen, dass es besser ist, sich nicht auf die grobsinnlichen Anschauungen über die Lage der Faktoren im Chromosom festzulegen, sondern MoRgans diagrammatische Darstellung nur als eine geometrische Ausdrucksform für Kräfterelationen zu nehmen, deren Wesen zukünftiger Erkenntnis vorbehalten bleibt.

\section{IV}

Ist die Möglichkeit einer Erkenntnis dieser unbekannten Kräfte nun etwas in unendlicher Ferne liegendes? Ich glaube nicht. Und damit begeben wir uns num auch auf den Weg der Hypothese. Wenn wir auch noch keine definitive Kenntnis darüber besitzen, was die Erbfaktoren darstellen, so machen eine Reihe neuer Arbeiten (z.B. Loeb w. ChamBERLIN I9I 5, ONSLOW I9I 5, GoLDSCHMIDT I9I6) es wahrscheinlich, dass sie etwas mit der Gruppe der Enzyme zu tun haben. Nun ist es ja eine unter Morphologen weitverbreitete Anschauung, dass Erbsubstanz chemisch mit Chromatin identisch sei. Ich habe dies, angesichts der cytologischen Tatsachen über die Umwandlungen des Chromatins in den Geschlechtszellen nie recht glauben können. Und auch die physiologischen Chemiker haben gewichtige Gründe dagegen. Nun findet man aber in der Fermentliteratur die Tatsache verzeichnet, dass einmal die 
Enzyme besser Schädigungen widerstehen in Gegenwart von Nucleoproteiden, sodann, dass Oxydasen ebenso wie hydrolytische Fermente oft an Nukleoproteide gebunden sind. So liegt der Gedanke nahe, dass die Aufgabe des Chromatins es ist, die Vererbungsenzyme zu adsorbieren, als ihr Skelet zu dienen. ${ }^{1}$ Sollten nicht vielleicht hinter den Variabeln und Konstanten des Crossing over die Variabeln und Konstanten der Adsorptionsgesetze stecken? Schon eine oberflächliche Orientierung in diesem so anziehenden Gebiet (s. etwa die Bücher von BAyLiss I9II, Bechhold igi2, Michaelis igo9, Cohnheim igi2, Euler-Pope I9I2) zeigt, wie aussichtsvoll eine eingehende Betrachtung dieser Beziehungen ist. Es sei nur erwähnt, welches Licht eine derartige Betrachtungsweise auf die typischen Oberflächenwandlungen des Chromatins während der Synapsis und Wachstumsperiode werfen könnte. Und der Weg hier weiterzukommen ist sicher ein sehr einfacher. Exacte Untersuchungen über den Einfluss verschiedener Temperaturen auf die Crossoverzahlen könnten vielleicht schon die entscheidenden Werte liefern.

Osborne Zoological Laboratory

Yale University.

\section{CITIERTE LITERATUR}

BAYLISs, W. M., I9II The nature of enzyme action. 2. ed. ix +90 pp. London: Longmans, Green \& Co.

Bechнold, H., I9I2 Die Kolloide in Biologie und Medizin. xii $+44 \mathrm{I}$ pp. Dresden: T. Steinkopff.

Сohnheim, O., I9I2 Enzymes. Six lectures. viii + i73 pp. New York: J. Wiley \& Sons.

Euler, H., und Pope, T. H., IgI2 General chemistry of the enzymes, ix $+323 \mathrm{pp}$. New York: J. Wiley \& Sons.

Goldschmidt, R., 19I I Einführung in die Vererbungswissenschaft. Leipzig: W. Engelmann.

1913 Desgl. 2. Aufl. pp. xii +546 .

1914 in Correns-Goldschmidt, Vererbung und Bestimmung des Geschlechts. viii +189 pp. Berlin: Gebr. Bornträger.

I9I6 Genetic factors and enzyme reaction. Science N. S. 43:98-100.

Loeb, J., and Chamberlain, M. M., 1915 An attempt at a physico-chemical explanation of certain groups of fluctuating variation. Jour. Exp. Zoöl. $19: 550-568$.

${ }^{1}$ Ich hatte vor mehr als einem Jahr das Vergnügen, diese hier in wenigen Worten angedeuteten Anschauungen über die chemische Basis der Vererbung A. P. Miathews auszuführen. Worauf er mich darauf hinwies, dass er genau die gleiche Anschauung in einem im Drucke befindlichen Werk ausgesprochen habe. Dies Buch (Physiological Chemistry, New York, 1915) ist inzwischen erschienen und enthält p. I75-182 die betreffenden sehr interessanten Ausführungen. 
Michaelis, L., I909 Dynamik der Oberflächen. Dresden.

Morgan, T. H., Sturtevant, A. H., Mulder, H. J., und Bridges, C. B., Igis The mechanism of Mendelian heredity. xiii +262 pp. New York: Henry Holt $\&$ Co.

Muller, H. J., Igr6 The mechanism of crossing over. Amer. Nat. 50 : 193-221, 284$305,350-366,421-434$.

OnsLow, H., rgI5 A contribution to our knowledge of the chemistry of coat-color in animals and of dominant and recessive whiteness. Proc. R. Soc. London $609: 36-56$.

Stomps, T. J., I9I2 Mutation bei Oenothera biennis L. Biol. Centralbl. 32:521-535.

Sturtevant, A. H., I9r 3 The linear arrangement of six sex-linked factors in Drosophila as shown by their mode of association. Jour. Exp. Zoöl. 14:43-59. 